\title{
Myo-inositol supplementation as a preventive consideration for gestational diabetes mellitus
}

\section{George Asimakopoulos, Panagiotis Antsaklis, Mariana Theodora, Michael Sindos, Alexandros Rodolakis, Dimitrios Loutradis, George Daskalakis}

$1^{\text {st }}$ Department of Obstetrics and Gynecology, National and Kapodistrian University of Athens, "Alexandra" Hospital, Athens, Greece

\section{Corresponding Author}

$1^{\text {st }}$ Department of Obstetrics and Gynecology, National and Kapodistrian University of Athens, "Alexandra" Hospital, Athens, Greece, e-mail: asimakopoulos.geo5@gmail.com

\section{Abstract}

Gestational diabetes mellitus (GDM) is defined as impaired glucose tolerance with onset or first recognition during pregnancy, which is characterized by increased insulin resistance. GDM affects about 10\% of pregnancies and its prevalence is rising worldwide mainly due to the advancing maternal age and the increased prevalence of obesity. GDM is associated with pregnancy-related maternal and fetal morbidity (both antenatal and perinatal). Traditional treatments for the GDM include diet and, if it is necessary, insulin. However, the percentage of macrosomic newborns does not appear to be significantly reduced. Safe and effective preventive interventions are therefore, needed in an attempt to lower the incidence of gestational diabetes. Myo-inositol has been suggested to improve insulin resistance in women with insulin resistant syndromes such as GDM, polycystic ovarian syndrome, type 2 diabetes and metabolic syndrome. Several studies report a significant decrease in GDM incidence in women at risk for GDM comparing to the placebo group. However, the option of myo-inositol supplementation needs to be further explored as the current evidence is relatively limited.

Key Words: Myo-inositol - Gestational diabetes mellitus - Insulin resistance - Review

\section{Background}

Gestational diabetes mellitus (GDM) is defined as glucose intolerance with onset or first recognition during pregnancy, which is associated with increased insulin resistance ${ }^{1,2}$. This complication is associated with several risk factors such as advanced maternal age, increased body mass index (BMI) and family history or previous history of gestational diabetes ${ }^{2}$. The prevalence of GDM is rising worldwide mainly due to the advancing maternal age and the increased prevalence of obesity ${ }^{3}$. Although GDM is reported to affect about $10 \%$ of pregnancies, its prevalence is expected to be higher according to the newly pro- 
posed criteria for the diagnosis of $\mathrm{GDM}^{4}$.

Gestational diabetes mellitus is associated with higher risk of pregnancy complications. Perinatal morbidity is increased due to complications such as fetal macrosomia, shoulder dystocia, neonatal hypoglycemia and respiratory distress syndrome ${ }^{5}$. Maternal morbidity includes caesarean delivery, gestational hypertension, preeclampsia and predisposition to the development of type 2 diabetes (T2D) later in life ${ }^{6}$. Furthermore, the newborns with macrosomia are at increased risk for the onset of hypertension and diabetes later in life ${ }^{7}$.

Pregnancy is normally characterized by insulin resistance. Most women adjust during pregnancy and remain euglycemic by increasing insulin release. On the other hand, women who develop GDM are unable to counteract insulin resistance. Although insulin-sensitizing factors, such as metformin, were initially considered to be promising interventions for the prevention of GDM, they do not seem to be as efficacious as expected so far ${ }^{8}$. GDM is principally treated with diet and, if it is necessary, with insulin. The combination of intensive monitoring during pregnancy and the use of novel insulin regimens allows the effective regulation of blood glucose levels and improves the perinatal outcome. However, the percentage of macrosomic newborns does not appear to be significantly reduced (15-45\%) ${ }^{9}$.

Inositol is a sixfold alcohol of cyclohexane which is present in animal and plant cells ${ }^{10}$. Myo-inositol and d-chiro inositol are the most common isoforms of inositol in eukaryotic cells with insulin-mimetic properties and potential therapeutic effects on insulin-mediated diseases ${ }^{11,12}$. Myo-inositol is an intracellular insulin mediator that provides more available phosphatidylinositol. Phosphatidylinositol seems to be important in the relation of insulin with its receptor leading to increased insulin sensitivity ${ }^{13}$. Myo-inositol is present in many foods, such as cereals, corn, legumes and meat. It is synthesized principally by the liver.

Preventive strategies are considered to be better than active response following the onset of a complication. Safe and effective interventions are therefore, needed in an attempt to lower the incidence of gestational diabetes. Such interventions could also decrease the economic burden of the disease and benefit health care systems.

In the last years, several clinical studies have been conducted to explore the efficacy and safety of myoinositol use for the prevention of GDM.

\section{Myo-inositol and Gestational Diabetes Mellitus}

Several clinical studies have investigated the potential effect of dietary myo-inositol supplementation on the prevention of GDM in high-risk women.

In a retrospective study conducted in pregnant women affected by PCOS, D'Anna et al. ${ }^{14}$ observed that myo-inositol administration, throughout the pregnancy course, may reduce the prevalence of GDM. In particular, they demonstrated a lower prevalence of GDM in PCOS women who achieved conception on myo-inositol supplement and continued this regimen during pregnancy, compared with PCOS women who conceived on metformin and discontinued it after the diagnosis of pregnancy (17.4 vs. $54 \%, \mathrm{P}=0,001$ ).

Another open-label RCT ${ }^{15}$ reported a significant decrease of GDM incidence in non-obese women, with a family history of T2D, treated with myo-inositol and folic acid from the end of the first trimester throughout the remainder of the pregnancy compared with similar control subjects treated with folic acid alone ( 6 vs. $15.3 \%, \mathrm{P}=0.04$ ). Moreover, fetal macrosomia was significantly reduced ( 0 vs. $7 \%, P=0.007$ ) in the myo-inositol group and the mean birth weight was also significantly reduced in the treatment group (P $=0,018)$. The authors also reported a statistically significant decrease of fasting plasma glucose levels $(\mathrm{P}=0.001)$ and 1-h plasma glucose levels on the 75gr 
oral glucose tolerance test (OGTT) $(\mathrm{P}=0.02)$ in the myo-inositol group compared to the control group. The same authors ${ }^{16}$ reported a lower incidence of GDM (14 vs. $33.6 \%, P=0.001$ ) and a greater reduction in the homeostasis model assessment of insulin resistance ( $-1.0 \pm 3.1$ vs. $0.1 \pm 1.8, \mathrm{P}=0.048)$ among 99 obese pregnant women treated with myo-inositol and folic acid from the first trimester to delivery compared to 107 obese pregnant women treated with folic acid alone.

Matarelli et al. ${ }^{17}$ demonstrated that myo-inositol supplementation in pregnancy reduces the incidence of GDM in women at high risk of this disorder accompanied by improved outcomes. In particular, women treated with myo-inositol showed a significantly lower prevalence of GDM in mid-pregnancy $(R R=0,127,95 \%$ CI $[0.032-0.502], \mathrm{P}=0,001)$, required less insulin therapy $(R R=0.136,95 \% \mathrm{CI}$ [0.018-1.031], $\mathrm{P}=0.053)$, delivered at a later gestational age (95\% CI [-2.578 to -0.948$], \mathrm{P}=0.001$ ) and had significantly smaller babies (birth weight is expressed as percentiles, 95\% CI [10.807 - 30.116], $\mathrm{P}=0.001$ ) with fewer episodes of neonatal hypoglycemia $(R R=0.052,95 \%$ CI $[0.003-0.849], \mathrm{P}=$ 0.038) compared to placebo.

In a randomized controlled trial, Santamaria et al. ${ }^{18}$ reported a significant decrease of GDM incidence in overweight non-obese women (pre-pregnancy BMI $\geq 25$ and $<30$ ) treated with myo-inositol and folic acid from the end of the first trimester until delivery compared with similar control subjects treated with folic acid alone $(11,6$ vs. $27,4 \%, P=0.004)$.

In a meta-analysis of five trials containing 513 participants, Zheng et al. ${ }^{19}$ demonstrated that women treated with myo-inositol showed a significantly lower incidence of GDM ((RR 0.29; 95\% CI, 0.19-0.44; $P<0.00001)$ and a statistically significant decrease of fasting plasma glucose levels (MD, -0.36 ; 95\% CI, -0.51 to $-0.21 ; P<0.0001$ ), 1 -h plasma glucose levels (MD, $-0.63 ; 95 \% \mathrm{CI},-1.01$ to
-0.26; $P=0.002$ ) and 2-h plasma glucose levels (MD, $-0.45 ; 95 \% \mathrm{CI},-0.75$ to -0.16 ; $P=0.002$ ) on the $75 \mathrm{gr}$ oral glucose tolerance test (OGTT) compared to placebo. Moreover, women treated with myo-inositol had significantly smaller babies (MD, $-116.98 ; 95 \%$ CI, -208.87 to -25.09 ; $P=0.01$ ) compared to placebo.

In a systematic review of Cochrane Database, Crawford et al. ${ }^{20}$ reported a significantly lower incidence of GDM (RR $=0.43,95 \% \mathrm{CI}[0.29$ to 0.64 ]; three trials; $\mathrm{n}=502$ women) in women treated with myo-inositol compared to control subjects. However, no clear difference was reported in the risk of hypertensive disorders of pregnancy (average $\mathrm{RR}=0.43,95 \% \mathrm{CI}$ [0.02 to 8.41]; two trials; $\mathrm{n}=398$ women), caesarean section ( $\mathrm{RR}=0.95,95 \% \mathrm{CI}[0.76$ to 1.19]; two trials; $\mathrm{n}=398$ women), macrosomia (average $\mathrm{RR}=0.35,95 \% \mathrm{CI}$ [0.02 to 6.37]; two trials; $\mathrm{n}=398$ infants), neonatal hypoglycaemia ( $\mathrm{RR}=0.36$, 95\% CI [0.01 to 8.66]; two trials; $\mathrm{n}=398$ infants), shoulder dystocia (average $\mathrm{RR}=2.33,95 \% \mathrm{CI}[0.12$ to 44.30]; two trials; $n=398$ infants). There were no maternal adverse effects of therapy in the two trials that reported on this outcome. The authors concluded that dietary supplementation with myoinositol during pregnancy demonstrates a potential benefit for reducing the incidence of gestational diabetes. However, the current evidence is based on small trials conducted in Italy leading to concerns about the lack of generalizability of the evidence to other settings.

Another study conducted by Dell' Edera at al. ${ }^{21}$ demonstrated that the supplementation with D-chiroinositol and D-myo-inositol allows a better control of maternal glycemia resulting in a lower incidence of GDM (RR = 3.35; 95\% CI = 1.37-8.17; P = 0.0028) and more favorable perinatal outcomes referring to the risk of macrosomia $(\mathrm{RR}=5.12 ; 95 \% \mathrm{CI}=$ 1.21-21.68; $\mathrm{P}=0,0099$ ) in women with an elevated fasting glucose value (glycemia $\geq 5.1 \mathrm{mmol} / \mathrm{l}$ or 92 $\mathrm{mg} / \mathrm{dl}$ and $\leq 7.0 \mathrm{mmol} / \mathrm{l}$ or $126 \mathrm{mg} / \mathrm{dl}$ ). 
In a systematic review and meta-analysis of randomized controlled trials, Guo et al. ${ }^{22}$ reported that, compared with placebo, myo-inositol supplement is associated

with a reduced risk of developing GDM (RR = $0.44,95 \%$ CI [0.32, 0.62], $\mathrm{P}<0.0001)$, a significantly lower fasting OGTT (MD $=-0.18,95 \%$ CI $[-0.24$, $-0.12], \mathrm{P}<0.00001)$, a lower 1h OGTT (MD $=-0.55$, $95 \%$ CI $[-0.81,-0.28], \mathrm{P}<0.00001)$ and a lower $2 \mathrm{~h}$ OGTT (MD $=-0.58,95 \%$ CI [-0.94, -0.23], $\mathrm{P}=0.001$ ). Furthermore, myo-inositol supplementation was associated with a significantly lower incidence of pre-term delivery $(\mathrm{RR}=0.30,95 \% \mathrm{CI}[0.11,0.79]$, $P=0.01)$. However, no significant differences were reported in the incidence of other complications, including distress respiratory syndrome, macrosomia, shoulder dystocia, and neonatal hypoglycemia.

A secondary analysis from three randomized controlled trials conducted by Santamaria et al. ${ }^{23} \mathrm{dem}$ onstrated that myo-inositol supplementation in early pregnancy is associated with a reduced incidence of GDM and reduced risk of preterm birth and macrosomia in pregnant women who are at risk for GDM (obesity [pre-pregnancy body mass index $\geq 30 \mathrm{~kg} /$ $\mathrm{m} 2$ ], overweight [pre-pregnancy body mass index $\geq 25$ to $<30 \mathrm{~kg} / \mathrm{m} 2$ ], or parent affected by type 2 diabetes mellitus). In particular, myo-inositol treatment was associated with a significantly reduced GDM onset by $66 \%(\mathrm{OR}=0.34 ; \mathrm{P}<0.001)$ and improved fasting $(\mathrm{OR}=0.37 ; \mathrm{P}=0.001)$ and 2-hour glucose values $(\mathrm{OR}=0.44 ; \mathrm{P}=0.01)$. Moreover, women treated with myo-inositol showed a significantly decreased risk for preterm birth $(\mathrm{OR}=0.44 ; \mathrm{P}=0.03)$ and macrosomia (OR $=0.38 ; \mathrm{P}=0.04)$, and border line decreased values for $L G A(O R=0.52 ; \mathrm{P}=0.05)$ and gestational hypertension $(\mathrm{OR}=0.34 ; \mathrm{P}=0.06)$.

\section{Conclusion}

Myo-inositol supplementation seems to have some potential in preventing GDM. In particular, the daily administration of myo-inositol appears to improve the glycemic homeostasis and reduce GDM rate without the risk of side effects. Moreover myoinositol offers an option with good tolerability and good compliance. However, further studies including pregnant women of different ethnicities with varying risk factors should be encouraged. Due to the poor quality of the available evidence, further good-quality RCTs are still needed to confirm the effectiveness and the optimal use (dose, frequency, timing of administration) of myo-inositol for GDM prevention.

\section{References}

1. ACOG Practice Bulletin. Clinical management guidelines for obstetrician-gynecologists. Number 30, September 2001 (replaces Technical Bulletin Number 200, December 1994). Gestational diabetes. Obstetrics and gynecology 2001, 98(3):525-538.

2. Buchanan TA, Xiang AH: Gestational diabetes mellitus. The Journal of clinical investigation 2005, 115(3):485-491.

3. Albrecht SS, Kuklina EV, Bansil P, Jamieson DJ, Whiteman MK, Kourtis AP, Posner SF, Callaghan WM: Diabetes trends among delivery hospitalizations in the U.S., 1994-2004. Diabetes care 2010, 33(4):768-773.

4. Ryan EA: Diagnosing gestational diabetes. Diabetologia 2011, 54(3):480-486.

5. Metzger BE, Lowe LP, Dyer AR, Trimble ER, Chaovarindr U, Coustan DR, Hadden DR, McCance DR, Hod M, McIntyre HD et al: Hyperglycemia and adverse pregnancy outcomes. The New England journal of medicine 2008, 358(19):1991-2002.

6. International Association of Diabetes and Pregnancy Study Groups Recommendations on the Diagnosis and Classification of Hyperglycemia in Pregnancy. Diabetes care 2010, 33(3):676.

7. Szostak-Wegierek D, Szamotulska K: Fetal devel- 
opment and risk of cardiovascular diseases and diabetes type 2 in adult life. Medycyna wieku rozwojowego 2011, 15(3):203-215.

8. Vanky E, Stridsklev S, Heimstad R, Romundstad P, Skogoy K, Kleggetveit O, Hjelle S, von Brandis P, Eikeland T, Flo K et al: Metformin versus placebo from first trimester to delivery in polycystic ovary syndrome: a randomized, controlled multicenter study. The Journal of clinical endocrinology and metabolism 2010, 95(12):E448-455.

9. Kc K, Shakya S, Zhang H: Gestational diabetes mellitus and macrosomia: a literature review. Annals of nutrition \& metabolism 2015, 66 Suppl 2:14-20.

10. Pintaudi B, Di Vieste G, Bonomo M: The Effectiveness of Myo-Inositol and D-Chiro Inositol Treatment in Type 2 Diabetes. International Journal of Endocrinology 2016, 2016:9132052.

11. Nas K, Tuu L: A comparative study between myoinositol and metformin in the treatment of insulin-resistant women. European review for medical and pharmacological sciences 2017, 21(2 Suppl):77-82.

12. Noventa M, Vitagliano A, Quaranta M, Borgato S, Abdulrahim B, Gizzo S: Preventive and Therapeutic Role of Dietary Inositol Supplementation in Periconceptional Period and During Pregnancy: A Summary of Evidences and Future Applications. Reproductive sciences (Thousand Oaks, Calif) 2016, 23(3):278-288.

13. Coustan DR: Can a dietary supplement prevent gestational diabetes mellitus? Diabetes care 2013, 36(4):777-779.

14. D’Anna R, Di Benedetto V, Rizzo P, Raffone E, Interdonato ML, Corrado F, Di Benedetto A: Myoinositol may prevent gestational diabetes in PCOS women. Gynecological endocrinology : the official journal of the International Society of Gynecological Endocrinology 2012, 28(6):440-442.

15. D'Anna R, Scilipoti A, Giordano D, Caruso C, Canna- ta ML, Interdonato ML, Corrado F, Di Benedetto A: myo-Inositol supplementation and onset of gestational diabetes mellitus in pregnant women with a family history of type 2 diabetes: a prospective, randomized, placebo-controlled study. Diabetes care 2013, 36(4):854-857.

16. D’Anna R, Di Benedetto A, Scilipoti A, Santamaria $A$, Interdonato $M L$, Petrella E, Neri I, Pintaudi B, Corrado F, Facchinetti F: Myo-inositol Supplementation for Prevention of Gestational Diabetes in Obese Pregnant Women: A Randomized Controlled Trial. Obstetrics and gynecology 2015, 126(2):310-315.

17. Matarrelli B, Vitacolonna E, D’Angelo M, Pavone G, Mattei PA, Liberati M, Celentano C: Effect of dietary myo-inositol supplementation in pregnancy on the incidence of maternal gestational diabetes mellitus and fetal outcomes: a randomized controlled trial. The journal of maternal-fetal \& neonatal medicine : the official journal of the European Association of Perinatal Medicine, the Federation of Asia and Oceania Perinatal Societies, the International Society of Perinatal Obstet 2013, 26(10):967-972.

18. Santamaria A, Di Benedetto A, Petrella E, Pintaudi B, Corrado F, D’Anna R, Neri I, Facchinetti F: Myoinositol may prevent gestational diabetes onset in overweight women: a randomized, controlled trial. The journal of maternal-fetal \& neonatal medicine : the official journal of the European Association of Perinatal Medicine, the Federation of Asia and Oceania Perinatal Societies, the International Society of Perinatal Obstet 2016, 29(19):3234-3237.

19. Zheng X, Liu Z, Zhang Y, Lin Y, Song J, Zheng L, Lin S: Relationship Between Myo-Inositol Supplementary and Gestational Diabetes Mellitus: A Meta-Analysis. Medicine 2015, 94(42):e1604.

20. Crawford TJ, Crowther CA, Alsweiler J, Brown J: Antenatal dietary supplementation with myo-ino- 
sitol in women during pregnancy for preventing gestational diabetes. The Cochrane database of systematic reviews 2015(12):Cd011507.

21. Dell'Edera D, Sarlo F, Allegretti A, Simone F, Lupo MG, Epifania AA: The influence of D-chiro-inositol and D-myo-inositol in pregnant women with glucose intolerance. Biomedical Reports 2017, $7(2): 169-172$.

22. Guo X, Guo S, Miao Z, Li Z, Zhang H: Myo-inositol lowers the risk of developing gestational diabetic mellitus in pregnancies: A systematic review and meta-analysis of randomized controlled trials with trial sequential analysis. Journal of diabetes and its complications 2018, 32(3):342-348.
23. Santamaria A, Alibrandi A, Di Benedetto A, Pintaudi B, Corrado F, Facchinetti F, D'Anna R: Clinical and metabolic outcomes in pregnant women at risk for gestational diabetes mellitus supplemented with myo-inositol: a secondary analysis from 3 RCTs. American journal of obstetrics and gynecology 2018, 219(3):300.e301-300.e306.

Received 28-11-2019

Revised 3-12-2019

Accepted 6-12-2019 